\title{
Current Situation and Prospect of Grassland Management Decision Support Systems in China
}

\author{
Qingwei Duan, Xiaoping Xin*, Guixia Yang, Baorui Chen, Hongbin Zhang, \\ Yuchun Yan, Xu Wang, Baohui Zhang, and Gang Li
}

\footnotetext{
${ }^{1}$ Hulunber GrassLand Ecosystem Observation and Research Station, Beijing 100081, P.R. China

${ }^{2}$ Key Laboratory of Resource Remote Sensing and Digital Agriculture, Ministry of Agriculture, Beijing 100081, P.R. China

${ }^{3}$ Institute of Agricultural Resources and Regional Planning, Chinese Academy of Agricultural Sciences, Beijing 100081, P.R. China xinxp@sina.com, Duanqingwei@ires.cn
}

\begin{abstract}
China, with wide grassland areas of the second rank throughout the world, is faced with a severe challenge on how to manage its vast and degenerating/degenerated grassland. Computer and network technologies are more and more widely applied in grassland production, research and education, which is just a greatly encouraging field. Tremendous achievements have been made in grassland management decision support system (GMDSS) research in developed countries at present, but there is still a long way to obtain a great development for developing countries, such as China. This paper reviewed the research progress and current situation in the GMDSS research and application in China. Concept models and empirical models are still hugely focused on the corresponding research fields in China, but the integrated GMDSS has not been well developed. Therefore, Chinese scientists must develop the integrated models from the existing models, and accordingly sinicize the GMDSS of models used in the developed countries for availably application. In the other hand, there is a same direction of research and development about the GMDSS not only for developed countries but also for China, which is going to be combined with internet, 3S (GIS, RS and GPS) and virtual technology.
\end{abstract}

\section{Introduction}

Information technology, digital agricultural technology and decision support systems (DSS) and other technologies have been put on a very important position in agriculture, but China is still relatively lagging behind in the field of grassland management DSS(GMDSS) especially when compared with the devoloped countries.In this serious international situation, we must catch up, otherwise we will not only lag behind in scientific research, but also prevents China to an inferior position in the international competition in the livestock industry.

\footnotetext{
* Corresponding author.
} 
The grazing system is a complex systems, including animals, plants, environment and peoples. About pasture growth, animal nutrition, the environment changes and the management of human behavior factors on grazing system interaction, a great deal of statistics models, mechanism models and simulation models have been set up in the past. Scientists established many computer DSS with the existing models, which improved the grassland management from a extensive management to a targeted intensive management. By simulating various production management strategy with the DSS, the simulation results can guide the production and management. Judging from the production level of GMDSS, it reducing the production management of blindness, reduce business risks; from the policy level, it helping the decision support for the government. With scientific and technological development and social needs increasing, grazing management simulation model have been made remarkable achievements in research and applications (Duan Qingwei, 2009).

This paper reviews the recent studies of domestic progress about GMDSS, including the base model, small DSS, network technology, remote sensing, GIS technology, WebGIS technology and virtual reality technology in DSS applications, and highlighted several ongoing large-scale domestic DSS research, and to outlook China's future trends in DSS research for reference of China GMDSS research and applications.

\section{Construction of Basic Model}

\subsection{Crop Growth Models}

Crop growth models can be used in pasture systems, hence the crop growth model would greatly facilitate pasture models study. Although the crop growth simulation research started very late, some basic researchs have been undertaken in China.Such as, "Rice population computer simulation model of material production" from Shanghai Institute of Plant Physiology of the CAS. "Rice growth calendar model (RICAM)" from Jiangxi Agricultural University; After 1990s, Institute of Agricultural Meteorology, Chinese Academy of Agricultural Sciences, finished CERES - Maize model Chinesization. In 1992, Jiangsu Academy of Agricultural Sciences finished "Rice cultivation Computer Simulation Optimization Decision System (RCSODS); Chinese Academy of Agricultural Sciences finished "Cotton Production Management Simulation System" (Zhang Wei, et al.). Liu Yilian, Chou Ziming (1994a) established populations of Leymus chinensis meadow Hulunbeier growth and yield simulation model based on the theory of crop growth simulation, combined with the growth characteristics of natural grass. The model consists of three sub-models: (1) photosynthesis and respiration; (2) assimilate distribution; (3) leaf area. The model can simulate aboveground biomass of Leymus chinensis populations with temporal dynamics by using the measured data validation of the model, and more objectively reflect the weather conditions on the grass growth and yield formation. Liu Yilian, Chou Ziming (1994b) carried on a certain numerical experimentation using the kinetic simulator model of Leymus chinensis. They discussed the influence of the meteorological factor-temperature, soil moisture, solar radiation not in the same year to change-to Leymus chinensis population output; The output calculates the dynamic 
"actual production", Which shows the statistical model simulation potential and the limitation. Pu erciren, Liu Naizhuang(1994) took the crops growth analog theory as a foundation, considered the multi-populations character of the steppe community, established the population biomass time dynamic synthesis influence's whole pattern for the high and cold Kobresia humilis meadow, Which can reflect effect of the meteorological factor and the biotic factor to biomass production, including random population physiological process-photosynthesis, respiration, assimilation products assignment- quantity simulations and the leaf area index dynamics, the community structure kinetic simulator et.al, 6 kinds of subschemata, which can quantify the grassland community ground biomass and the underground biomass seasonal variation, and that is a good attempt of prairie vegetation photosynthesis output simulator study. Wei Yurong (2004) used the Xilingol typical grassland animal husbandry meteorology data, from the environment factor to the forage and domestic animal influence's angle embarking, from qualitative to the quantitative analysis external environment condition to the grassland animal husbandry production's influence and the revelation lawn animal husbandry production rule, established a set decision-making service system to be possible to used in instructing the animal husbandry production.

\subsection{Remote Sensing Model Grassland Production}

A large number of grassland productivity models for livestock balance in China have also been established. Most of these models are fitted empirical models, which fitted between vegetation indices (such as NDVI, EVI et.al) obtained through remote sensing technology and ground biomass data.

Huang Jingfeng et.al (2000) established spectrum of natural pasture vegetation index and satellite remote sensing monitoring model, meteorological monitoring model, using spectral observations of natural pasture, forage production data, meteorological data and NOAA /AVHRR data, which can provide a timely and accurate scientific means to grasp the grassland production change.Xiong (2004) estimated global primary productivity (NPP)by using Application of Advanced Earth Observing Satellite II (Advanced Earth Observing satellite II, ADEOSII) of the global multi-spectral data. And he also produced a ground-NPP algorithm with temperate plants in Japan.Based on the algorithm, he estimated NPP pattern based on Mongolian Plateau decomposition vegetation index(VIPD). Liu Aihua, Xing Qi(2003) established a model with vegetation index and ground-based data using MODIS images, and monitored productivity of natural grasslands in Inner Mongolia.They estimated the natural grassland carrying capacity in the warm and cold season for the administrative departments at all levels grassland and livestock production based on macro-management decision-making. Li Bo, Shi Peijun, et.al. (1995) studied the principles and methods of grazing livestock balance dynamic monitoring system in the temperate grassland, designed the dynamic monitoring database,technology systems and remote sensing production dynamic monitoring model in order to establish wide range of dynamic monitoring system of grassland resources, and provide a basis for the grassland management decisions. Liu Xingyuan et.al (2010) established remote sensing monitoring model between the grass amount of biomass and edible forage in Gannan from 2005 to 2007 using daily MODIS surface reflectance product MOD09GA and 
fresh weight of aboveground biomass of the measured data and the actual stocking rate.and estabished a different season grazing pasture livestock balance of theoretical stocking rate models and monitoring model to analyze the productivity of natural grass and stocking density in space-time dynamic balance condition and regulation strategies.

\subsection{The Model Construction and Application of Mathematical Methods in Grassland Management}

System dynamics theory, gray relational grade analysis theory and gray forecast technique have some applications in grassland management.

Shan Baoyan, Xu Jianhua (1995) established the grassland ecological economic system optimal model of sustainable development, including pasture management and herd optimal control model of management optimization model,with which to explore the improvement of the capacity expansion of the system environment strategy.Yan Wenbin and Wang Xuemeng establish the dynamic model with the accumulated data of his grass-rabbit system.They put forward the optimization of artificial grassland ecosystem control strategy by using the gray system approach (Xu Jianhua, 2006).

\subsection{Systems Dynamics Simulation of the Grassland Management}

System dynamics is a discipline about the analysis of information feedback system, and also an integrated cross-discipline which to be used to understand system problems and resolve system problems.It is an integrated natural and social sciences disciplines based on systems theory,control theory, information theory,. Lv Shengli use system dynamics to simulate grassland ecosystem very early 1990s in China.

Lv Shengli, in Gansu Academy of Sciences, built a set of models in second production in micro and macro aspects of grassland.LvShengli (1991) studied"China grassland productivity simulation". He simulated the productivity of grassland for macro-and long-term dynamics by using system dynamics approach, system dynamics modeling and computer simulation tools. And he also made a scientific prediction and verification for the grassland productivity and the development trend of China. Outstanding features of this study compared with similar studies abroad are: 1), According to existing problems of grazing management in China, highlighting the breed of livestocks and livestock structure adjustment; 2), Focus on solving the contradiction between supply and demand cool-season forage, so that Grasssland Seasonal Livestock Manegement theory can reach the stage of dynamic simulation; 3 ), Suitable for medium and long term macro-policy research.

Lv Xiaoying, LvShengli (2003) established a dynamic model of grassland animal husbandry sustainable development using principles and methods of System Dynamics.This study simulated the livestock husbandry prospects for the future development on the 2000-2020 period in the northern temperate zone and alpine grassland steppe grassland.Dynamic model of sustainable agriculture development takes cold season and warm season forage needs satisfaction rate as bonds, which make primary pasture production and secondary livestock production process a organic whole coupled with the dynamic and complex feedback structure. With this model we 
can achieved analysis and comparison of a variety of sustainable agriculture development simulation programs, from which to reveal the barriers in the development process, seek strategies to improve the status of a favorable way, show various scenarios of possible development trends, provide a basis for policy-makers.

Aim at Gannan grassland animal husbandry practical situation, one of the major pastoral areas in Gansu Province, Duan Shunshan, Lv Shengli et.al (1995) analyzed the Gannan grassland animal husbandry development of power and constraints; predicted 30 years of pastoral animal husbandry in Gannan (1990-2020) the dynamic process of development and change; established long-term development of grassland animal husbandry in Gannan dynamic model by using system dynamics simulation technology, system dynamics flow diagram and spreadsheet functions.

\section{Construction of GMDSS}

\subsection{Grass-Livestock Digital Management DSS in Pastoral Area}

Lanzhou University preside over "the pastoral area grass livestock digitization to manage DSS" the topic (2007AA10Z232) from the People's Republic of China Department of Technical Science, Which was authorized in 2007. It is a special topic--modern agriculture area of technology digit agricultural technology-in the project the national high-tech research development plan (863 plans).This beginning and end age limit of the topic is August, 2007 to October, 2010, Beijing Raising livestock Research institute of Chinese Agriculture Academy of science is the cooperation unit. This topic sets an experiment area of the Gannan pastoral area, through the investigation and the monitoring to the grassland and the livestock husbandry, with the help of network techonology, 3S technology, system model and optimized decision-making, the exploration research based on MODIS.

(Moderate Resolution Imaging Spectradiometer) grassland remote sensing monitoring and the evaluating indicator system, they established the grassland information fast gain model, the grass livestock dynamical equilibrium and the analysis diagnostic model and the pastoral area grassland animal husbandry integrated management decision model.They made a contrastive analysis for lately 30 year grass livestock spatial variation condition, optimized grass livestock production technology flow, constructed GMDSS based on the network technology. This project already applied for the pastoral area grass-livestock digitizatal management DSS1.0 software copyright (the registration number: 2009SR043117, copyright owner (nationality): Lanzhou University: China. For the first time publication date: 2009-04-20, registration authorization date: 2009-09-28). Following research work is still in advance.

\subsection{Integrated Application of 3s Technology}

Yuan Qing et.al.(2006) take the multi- dimensional scientific data (spatial data, attribute data) and the expert knowledge as a foundation, take the modern $3 \mathrm{~S}$ technology as the support, establishes the Chinese grassland ecological environment monitoring and the appraisal information system. This system based on Internet, 
remote sensing technique and WebGIS and so on, and designed the system structure (to see Figure.1) to plan and to design the following content: 1) Basic data collection and reorganization (including science, society, economy, expert knowledge etc) ; 2) Spatial analysis and operation strategy and method study with the support of expert knowledge library; 3) Establishment of various multi- dimensional mathematical models; 4) Special- purpose geographic information system's design, development; 5) Design and development of computer auxiliary decision-making subsystem. The completed system has following functions: 1) Present situation accessment of grassland ecological environment (health, stable, productive forces, grass livestock balanced and so on); 2) Grassland ecosystem's change trend analysis in space and time sequence; 3) Computer auxiliary decisions-making of ecological rotection, storation, construction etc. This system not only may provide the theory basis for the national macroscopic decision-making, simultaneously provide the government department and the production unit with scienctific, reliable, timely background data and the dynamic change data, moreover benefit the ecological construction and the livestock husbandry production, the maintenance or restores the natural pasture in the ecological equilibrium state and continues forever to be used, have an important directio value in enhancing our country grassland scientific style, modernization, the informationization management level.

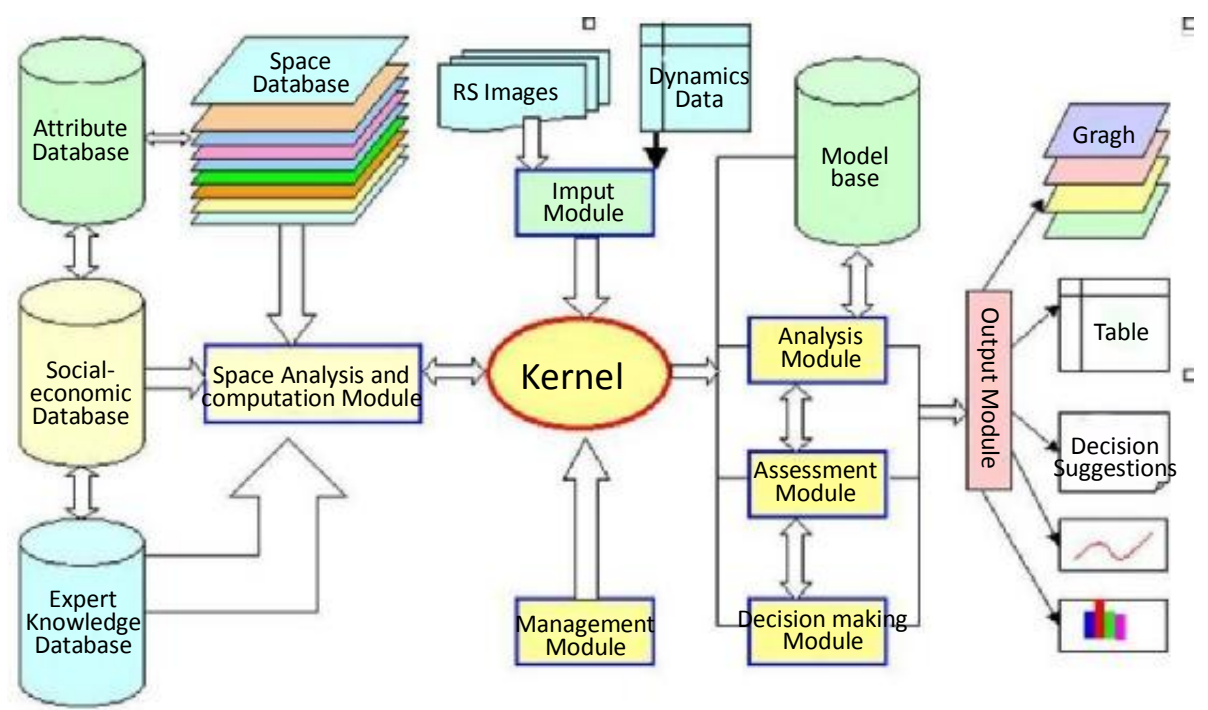

Fig. 1. Grassland ecological environment monitoring \&appraisal diagram under 3 s technical support

\subsection{Expert System of Prataculture Ecological Construction and Development}

Gansu grassland ecology research institute has developed "the Gansu Province Expert system of prataculture ecological construction and development" in October, 2001. 
This system take the $3 \mathrm{~S}$ technology and the internet as the development foundation, which can carry on point inquiry dough and polygon inquiry, including-"in where", "is what","plant what","how to grow"--four questions. While point inquiry dough and polygon inquiry take point (approximately $1 \mathrm{~km}^{2}$ ), township or county as the unit, which is enormous convenient for users. Main deficiencies of this system are: 1) The forage compatible research is carried on in the foundation of the grassland synthesis order classification retrieval chart,which only involves the compatibility of forage grass, not solve the forage planting and the variety compatible problem; 2) 56 kind of fine forage grass cultivation compatible chart in the system are in the investigation foundation, which is connected to spatial databases's field name, not inference results(Sun Juan, 2004). Afterward, by Lanzhou University grass farm science and technology institute Professor Chen Quangong, Ren Jizhou and Wang Jiayi (Lanzhou Gaobo computer information system engineering company senior engineer) etc completes WEST special project of the Science and Technology Department of China"Expert system of Chinese prataculture development and ecological construction" (2003BA901A20).The compact disc version is published at January, 2006. The Chinese prataculture and ecology" website based on WebGIS also opens afterward. The compact disc and the website are developed in the scientific discipline knowledge system of geoscience, agronomy and prataculture science, with $3 \mathrm{~S}$ technology, computer and information technology. They combinethe spatial databases, more than 2100 graph images, 2 million words, 2GB system capacity , and which have retrieval, inference,demonstration, printing function; contain 23 spatial databases of the national county level administration regionalization and the national various counties remote sensing images, grassland type, soil type, cultivation forage distribution,domestic animal distribution,agriculture and husbandry resources environment specifically and so on; Contains the Chinese prataculture and ecology,prataculture knowledge library, key area of cultivating forage, the grassland management and the ecological construction, plant disease diagnoses, the prataculture science and technology, the forage growth simulation, the prataculture information, various provinces prataculture and ecology etc 12 groups of 38 main modules. The household user with simple computer operation knowledge is possible to use this software and the website inquires.Both can reply accurately "in where", demonstration space position and correlational dependence and so on; "is anything", demonstration environment, agricultural resource, native vegetation and so on; "plants anything", demonstrated that suitable cultivation the forage type and recommends the improved seed; "how to plant", demonstration cultivation managerial technique and sickness, insect, rat damage and weed's prevention method; "raises what livestock breed", demonstrated fine livestock and livestock and poultry type and historical evolution; "how to raise", demonstrates the science raising method of various poultry variety. Several hundred agriculture colleges and universities and the Scientific research Unit in China, more than 2300 counties may obtain "Expert system of Chinese prataculture development and the ecological construction" the software compact disc (ISBN 7-89496-798-X) freely, users may also register "the Chinese grass industry and the ecology" the website (www.ecograss.com.cn) glance over (Lanzhou University news net, 2006). 


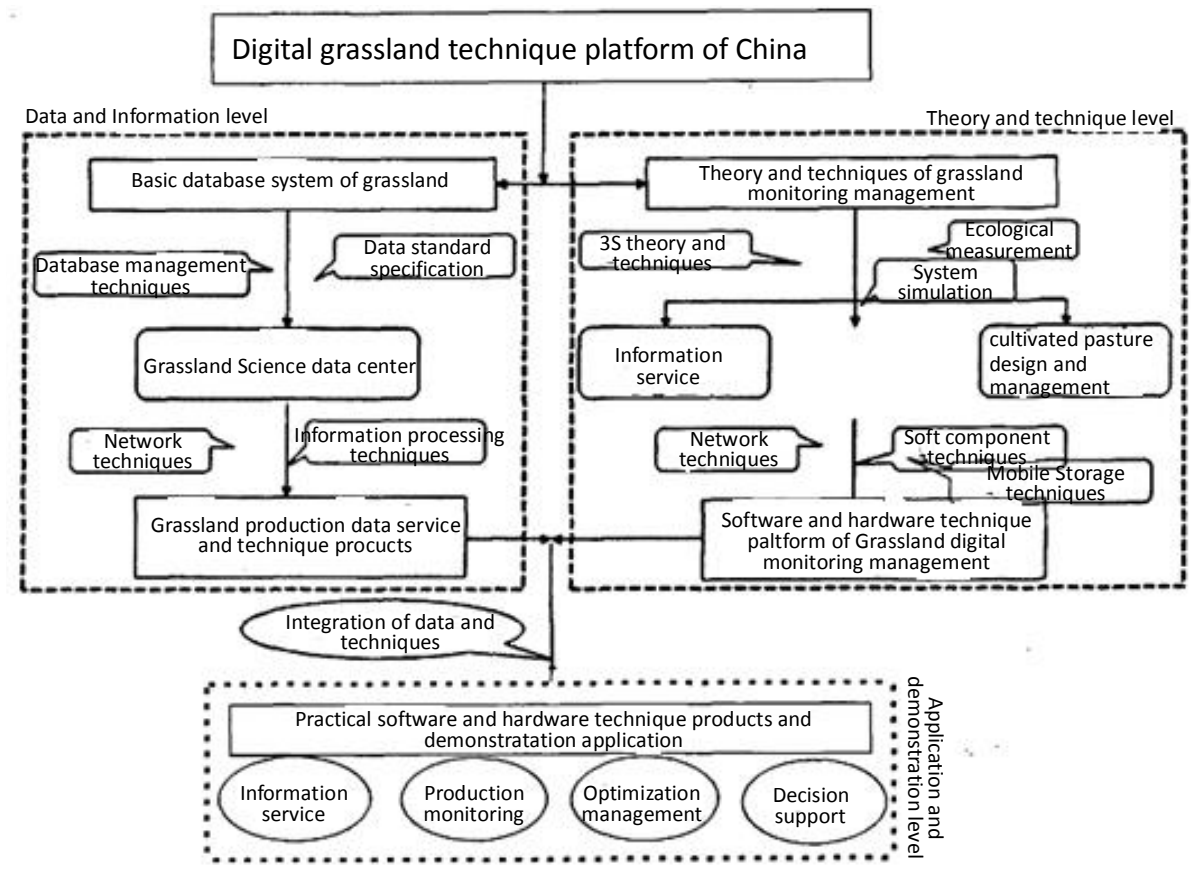

Fig. 2. Framework for the digital prataculture technique platform of China

\subsection{Chinese Digital Prataculture Theory Technology Platform}

The grassland management and the decision-making are a huge system, which need a great deal of data and technical support. Based on this reason, Institute of Agricultural resources and regional planning, Chinese Academy of Agricultural of Sciences (IARRP-CAAS) has constructed the huge digital pratacultural technology theory platform, which build the foundation for the Chinese prataculturae sustainable development. The establishment thinking of the "digital theory and technology platform of Prataculture" is using information technology and its latest research results, and prataculture management theory, and exploring critical technology issues and related to scientific assumptions of the prataculture digital monitoring and management, from which extracts China digital grassland theory and technology infrastructure, they are summed up as three levels :Prataculture data and information, the core theory and technology, application and demonstration (see Figure. 2).

\section{Small Decision Support Systems}

Small DSS is relatively more than big one in the farm management,such as pig farm, cattle farm's small DSS.The small DSS can realize the industrialization and the automatic control, to be quite convenient with the market trail connection, compared 
with the huge grassland area livestock husbandry. And small DSS is manoeuvring, therefore develop rapidly.

Xiong Benhai (2005) has constructed "the cow fine cultivation technology platform" using RFID, PDA and wireless local network technologies, which realized the comprehensive gathering cow's individual body situation information and through calculate gain cow's date nutrition required quantity using the nutrient forecast model and the cow secretes curve model, which directs the instruction date grain formula, achieves the cow individual the fine raising goal. Wang Zhonghua (2005) uses digital technique collecting cow milk production quantity, young ingredient, amount of exercise, body situation, body weight change information, as well as cow's foundation date grain picks the appetite, through the overall system automatically controls feed's supplement plan, which realized cow nutrition need, feed design, information acquisition, cow automatic diagnosis, automatic control et al various technological means.

Expert system of the alfalfa planting management and pest control developed by Beijing Intelligence Valley Science and Technology Limited Company can be used to alfalfa planting management and pest control production. (National Copyright Administration registration number: 200210952; Software copyright registration number: 2002SR2425; Patent of invention number: 02121283.X) (Bai Fengshuang, 2003).

Jiang Wenlan has set up a big sustainable production model of forage resources population protection using the biocybernetics method, and take the experiment as an example, she had determined the concrete protection target and the grazing management measures, studied the unicast artifiicial pasture optimum control model with herd the countermeasure; constructed the artifiicial pasture applying fertilizer model and the grazing system nutrient cycle mechanism model, and proposed "Integrated computer fertilizer recommendation expert system for artificial pasture".

\section{Calibration and Application of Broad Model}

Although the overseas software's chinization is not a long-term plan, but is also an important direction. Through taking example by the overseas advanced technology can also promote the domestic DSS research to a certain extent.

Institute of Agricultural Resources and regional Planning,Chinese Acedamy of Agricultural Sciences (IARRP-CAAS) introduced and improves the Australian GMDSS-GRAZPLAN for North China grassland.They used long-term observation data of the temperate grassland, fitted and adjusted its first floor model parameter, based on which, they established a suite of domestic animal system's system simulation and the dynamic management system for Chinese temperate grassland, which could accurately simulate the ungrazing grassland production and the community structure dynamics and grassland production and the community structure under different stocking rates, defferent utilization pattern (rotational grazing or forbidden grazing)in grazing situation.which can provide references for the family pasture livestock production, ecology,economically optimized management and dynamically regulation (Tang Huajun, Xin Xiaoping, 2009). 


\section{New Technology Application in Grassland Management}

\subsection{Application of GIS and WebGIS Technology}

The model and the network bond is a current international agriculture model study important tendency. Expert system (ES) or DSS combine with GIS, whicn may cause ES or DSS decision-making process integrated with the geography information. Through useging GIS analysis result, we can enhance ES and DSS decision-making level. At the same time, decision-making result issuing through GIS may strengthen intuitive, and builds the foundation for the further localization implementation (Chen Liping, Zhao Chunjiang, 2002). Sun Juan (2004) established forage adaptation and returning cropland back to grassland DSS based on GIS, which acilitated Gansu Province forage seed selection for Gansu returning cropland back to grassland,that is offering decision support and basis for forage adaptation problem.

\subsection{Virtual Reality Technology}

The virtual reality technology is a technology of reflecting the biological growth process with graphs and dynamic images. Realizing the virtual reality in GMDSS will perform forage growing and animal development process clearly with the virtual plant and virtual animal, and visually manifest the influence of various factors to the forage growing and the animal development.But there is still no advance in virtual grassland in China.

Liu Shengping, Zhu Yeping (2008) have established the wheat analog modeling based on the agent technology. The agent technology involves system science, artificial intelligence, software engineering and pattern layout knowledge, and it may provide the available agent of soil, weather, irrigation, applying fertilizers, nitrogen equilibrium and water balance of the crops growth model. These agent also provide the sharing codes for re-development of the other crops models.

\subsection{PDA}

PDA is a Personal Digital Assistant,a Pocket PC.PDA Is as the name suggests that is auxiliary individual work as a digital tool, facilitates carrying, convenient for open country using. At present, domestic and foreign data acquisition software based on the PDA already carry on the practical application in forestry, crops applying fertilizer, cadastration, medical service and many other domains(Wu Shouzhong,2007), but PDA are quite few in related research of the grassland managerial data gathering aspect. Wu Shouzhong(2007) developed the PDA grassaland rat damage data acquisition system with $\mathrm{C \#}$ as the development kit and with technical metasyntheses of PDA, GPS, XML.He also developed application tool softwares including the spatial collection law, the settled spot collection law, the settled area collection and the metal pliers date gathering law, which realized the grassland mouse data acquisition semiautomatically, and changed the tradition field datacollection way, and raised the working efficiency(See Figure.3) 


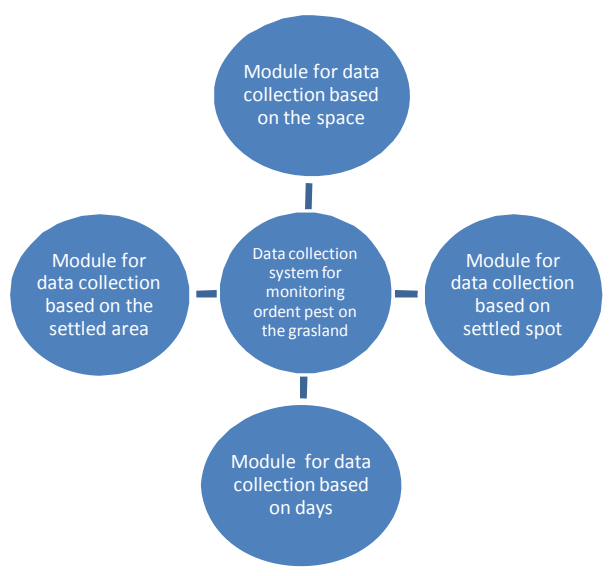

Fig. 3. Data collection system for monitoring rodent pest on the grassland based on PDA (Wu et al,2007)

\section{Prospects of Grassland Decision-Making System in China}

Study of GMDSS has made some achievements, such as grassland productivity model and grassland system simulation, but there is still no practical GMDSS in China.

In future research, we should firstly combine the existing resources, including the remote sensing monitoring data, the ground datum and the meteorological data etc, and full use of IT technology (for example,WebGIS technology). We should complete the forage adaption assessment and the ecology regional planning to direct macroscopic region forage production. Secondly, we must research and develop GMDSS of our own proprietary intellectual property rights and suit the Chinese grassland production management profitting from domestic and foreign research foundation;and strengthen mechanism model research.Moreover enhancing overseas cooperation with the developed countries, or adjusting overseas mature model is a convenient way.For example,the introduction of Australian GRAZPLAN system by IARRP-CAAS. Simultaneously we should realize combination between the remote sensing monitor data and DSS, and realize timeliness and effectiveness of GMDSS. China has approximately $4 \times 10^{8} \mathrm{hm}^{2}$ grassland, the area is no 2 in the world, we should develop our own GMDSS adapt to China grassland geography, climate and special grazing tactics, which is requested from the extensive economy to the intensive economy trend of development. We must realize the grazing management mathematically quantification and controllable; we must develop comprehensive GMDSS, as well as small grazing management decision-making calculator. Along with developement of computer network technology, small calculators may provide the online use. In brief, our country's grazing management is still the family pasture-like extensive management, There is still a very big disparity compared with the developed country. Our grazing management DSS research also just begin.Because the DSS research is time and expends-consuming work, usually, we need model research foundation, 
simultaneously need the massive data foundation and computer application ability, and the multi-disciplinary cooperation. We hope in the near future, we might realize dynamic grassland process management based on the knowledge library, the model base and the remote sensing database, and realize the virtual reality--the grassland management process and the effect present by the visual picture form in front of us.

Acknowledgment. Research was supported by the earmarked fund for Modern AgroIndustry Technology Research System, key scientific and technological project of Inner Mongolia(20091403), meteorology assessment technology of grassland ecology health from meteorology industry science and technology (GYHY200906029-2) and grassland carring capacity and livestock deploy in north China meadow grassland from industry science and technology of China Ministry of Agriculture (200903060).

\section{References}

[1] Xiong, B., Qian, P., Luo, Q., Lv, J.: Meticulous solution on livestock husbandry based on individual dairy cattle body conditions. In: Digital Agriculture Research Progress,Edited by general experts group of digital agricultural specialization of 863 plan, and National Engineering Research Center for Information Technology in Agriculture (NERCITA), September 2005, pp. 435-441. Chinese agricultural sciences and technology press, Beijing (2005)

[2] Wang, Z.: Function and system design of digital technology in the production of dairy cattle. In: Digital Agriculture Research Progress,Edited by general experts group of digital agricultural specialization of 863 plan, and Nationa 1 Engineering Research Center for Information Technology in Agriculture (NERCITA), September 2005, pp. 452-454. Chinese agricultural sciences and technology press, Beijing (2005)

[3] Huang, J., Wang, X., Wang, R., Hu, X.: A Study on Monitoring and Predicting Models of Grass Yield in Natural Grassland. Journal of Remote Sensing 1(5), 69-74 (2001)

[4] Yan, X.: Geospatial information science (Quarterly) 2(7),117-123 (2004)

[5] Liu, S., Zhu, Y.: Establish Wheat model based on Agent technology. In: Agriculture Information Technology and Management, Edited by Agricultural Information Institute of Chinese

[6] Zhang, W., Dong, Y., Zhang, Q., Yuan, J., Du, Q.: Trends Analysis of Domestic and international agricultural science and technology. Information Center Chinese Academy of Sciences, http: / / www. docin. com/p-31936733.html

[7] Digital pastoral livestock management decision support system, http: / / www. chinadigitalgrass.com/

[8] Wu, S., Gao, L., Shi, D., Su, H., Liang, J.: Development Data Collection System of the Rodent Pest on the Grassland Based on the PDA with GPS. Acta Agrestia Sinica 6(15), 550-555 (2007)

[9] Lv, S., Duan, S., Song, B.: Dynamics Model of Grassland Agricultural Ecosystem. Acta Prataculture Sinica 4 (1990)

[10] Lv, S., Song, B.: Dynamics Model of Grassland Second Production. Gansu Social Sciences (1991) (supplementary issue)

[11] Lv, S., Song, B.: Study on Micro Dynamics Model of Grassland Second Production. Forage and livestock, 4-10 (March 1992)

[12] Lv, X., Lv, S.: Sustainable Development of Grassland Livestock Husbandry in Main Pasturing Areas of China. Gansu Social Sciences, 115-123 (February 2003) 
[13] Duan, S., Zhang, W., Lv, S., Song, B.: The Dynamics Simulation of Development for Intermediate or Long Term on Gannan Grassland Animal Husbandry. Pratacultural Science (January 1995)

[14] Shan, B., Xu, J.: The Optimization Model for the Sustained Development of Grassland Eco-Economic System. Pratacultural Science 6(12), 9-13 (1995)

[15] Xu, J.: Mathematical Methods of Cotemporary Geography, 2nd edn. Higher Education Press, Beijing (June 2006)

[16] Liu, A., Qi, X., Gao, W., Yang, G., Su, R., Ha, S., An, M.: The monitor of productivity and the estimates of carring capacity of natural grassland in Inner Mongolia in 2003. Inner Mongolia Protaculture 15(4), 1-3 (2003)

[17] Li, B., Shi, P., Lin, X.: A research on the dynamic monitoring system for forage-livestock balance in the temperate grassland of China. Acta Agrestia Sinaca (2), 96-102 (1995)

[18] Lanzhou University News. Western Specialisation of Ministry of Science and Technology -China grass industry development and environmental protection expert system\&quot-has made significant progress (2006-04-13),

http://news. lzu.edu.cn/content/5310shtml

[19] Bai, F.: Improve alfafa cultivation technology by using informatization. In: Proceedings of Second China alfalfa Development Conference and grass seed, machinery, products exhibition (2003)

[20] Sun, J.: GIS-based forage adaptability in decision support system of returning cropland back to grassland. Gansu Agricultural University Doctoral Dissertation (2004)

[21] Chen, L., Zhao, C., Liu, X., et al.: Design and Implementation of Precision Agriculture Intelligent Decision Support System. Transactions of the Chinese Society of Agricultural Engineering 18(2), 145-148 (2002)

[22] Tang, H., Xin, X., Yang, G., et al.: Modern theory and technology of digital Grassland Research Progress and Prospects. Chinese Journal of Grassland 31(4), 1-8 (2009)

[23] Ping, X., Liu, N., Yuan, W., et al.: Biomass Allocation of Leymus Chinensis population:a dynamic simulation study. China Jounal of Applied Ecology 18(12), 2699-2704 (2007)

[24] Wei, Y.: Research on the Grassland Model and Balance Model of Livestock Capacity in Typical Grassland of China.China agricultural University Master's thesis (2004)

[25] Liu, Y., Chou, Z.: Dynamic simulation model of Leymus Chinensis development in Inner Mongolia meadow steppa. Chinese Agricultural Meterology 15(2), 43-46 (1994a)

[26] Liu, Y., Chou, Z.: Numerical experimentation of Dynamic simulation model of Leymus Chinensis development in Inner Mongolia meadow steppa. Chinese Agricultural Meterology 15(3), 21-24.32 (1994b)

[27] Ciren, P., Liu, N.: Dynamic simulation of grassland biomass. Chinese Journal of Ecological Agriculture (3) (1994)

[28] Liu, X., Feng, Q., Liang, T., Long, R.: Spatial-temporal Dynamic Balance between Livestock Carrying Capacity and Productivity of Rangeland in Gannan of Gansu Province, China. Chinese Journal of Grassland 32(1), 99-106 (2010)

[29] Yuan, Q., Wang, J., Zhang, D.: Grassland ecological environment monitoring and appraisal strategy discussion under $3 \mathrm{~S}$ technical support. Chinese Grass Academic society

[30] Sichuan, J.: Grassland resources and utilization specialized committee in 2006 5th national academic seminar collection. Materials for internal reference (2006)

[31] Guo, Y., Li, B.: Foundation of digital agriculture - virtual plant. In: Symposium of China Digital Agriculture and Rural Informationization Development Strategy, Ministry of Science and Technology Division of Rural and Social Development, pp. 53-59. China Agriculture Press, Beijing (2003) 